\title{
Pratiques
}

Linguistique, littérature, didactique

$165-166$ | 2015

Étudier les figures en contexte : quels enjeux ?

\section{Figures et oralité}

Figures and Orality

\section{Catherine Rouayrenc}

\section{(2) OpenEdition}

Journals

Édition électronique

URL : https://journals.openedition.org/pratiques/2527

DOI : 10.4000/pratiques. 2527

ISSN : 2425-2042

\section{Éditeur}

Centre de recherche sur les médiations (CREM)

Référence électronique

Catherine Rouayrenc, «Figures et oralité », Pratiques [En ligne], 165-166 | 2015, mis en ligne le 01 octobre 2015, consulté le 07 décembre 2022. URL : http://journals.openedition.org/pratiques/2527 ; DOI : https://doi.org/10.4000/pratiques.2527

Ce document a été généré automatiquement le 29 septembre 2020.

Tous droits réservés 


\title{
Figures et oralité
}

\author{
Figures and Orality
}

\author{
Catherine Rouayrenc
}

1 Je voudrais montrer, à travers les figures, comment l'oralité dans le roman, c'est-à-dire ce par quoi un texte a un caractère oral, a pu évoluer depuis le début du xxe siècle.

\section{Oralité et co(n)texte}

2 Quand, dans la seconde moitié du XIX ${ }^{\mathrm{e}}$ siècle, les Frères Goncourt se demandent «si ce qu'on appelle "les basses classes" n'avait pas droit au Roman ${ }^{1}$ ", le langage du roman est le langage littéraire et ce dans les dialogues aussi bien que dans la narration, même si quelques auteurs ont tenté précédemment de restituer la parole du peuple (Wolf, 1990 ; Philippe, Piat, 2009 : 57-64).

3 À moins que le cotexte n'oblige à y voir une simple marque de registre liée à la situation d'énonciation, donc d'oralité (Rouayrenc, 1988, 1994), toute irrégularité langagière par rapport à la norme prescriptive, la Norme (Bédard, Maurais, 1983 ; Le Français moderne, 1982), reconnue comme seule norme de référence, était reçue comme langage "populaire ». En témoigne la réception de Voyage au bout de la nuit (V) (1932) dont le langage est dit « populaire » par quasiment tous les critiques (Derval, 1993).

Depuis Céline, le parlé, du fait qu'il pénètre la narration, a changé de statut. Ce qui était perçu comme populaire ne l'est plus que comme langage oralisé. La notion d'oralité est donc une notion relative, liée au cotexte et au contexte sociolinguistique.

Cette évolution de perception est liée à une évolution progressive dans la relation entre langage de la narration et langage des dialogues. Jusqu'au début du xxe siècle, le langage non littéraire, tenu pour intrus dès lors qu'il entre dans la narration, est signalé par l'italique ou plus souvent par les guillemets. Certains écrivains ont recours aux deux, parfois indifféremment comme G. Chevallier dans La Peur (1930). Paradoxalement, quelques écrivains du début $\mathrm{du} \mathrm{xx}^{\mathrm{e}}$ siècle très soucieux de la Norme, tel Poulaille, ont fait de l'hypercorrection en utilisant des guillemets dans les dialogues : « - [...] J'ai vu la mère 
Martin [...] la "beurre et œufs" au marché [...].» (Le Pain quotidien [PQ], 1934). Paradoxalement encore, les guillemets peuvent subsister dans la narration, même quand l'oral y pénètre. On en trouve ainsi dans les premiers romans de Céline : "Derrière nous, le "beurre et œufs" arrêtait pas de ramener sa cerise » (Mort à crédit, 1936).

\section{Les figures de l'oralité}

6 La notion de figure, quand il s'agit d'oral, ne va pas sans poser de questions. Selon C. C. Du Marsais $(1977: 11):$ « Hors un petit nombre de figures semblables, réservées pour le style élevé, les autres se trouvent tous les jours dans le style le plus simple, et dans le langage le plus commun ». Cette banalité n'est-elle pas en contradiction avec une définition récente, telle que celle de M. Bonhomme (2005: 39) : «Produire une figure, c'est engendrer une

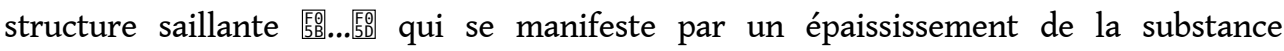
langagière dans certaines séquences discursives ».

7 Comme le remarque C. C. Du Marsais, l'oral quotidien, et de ce fait l'oralité, semble exclure en principe certaines figures, notamment les figures de quelque extension, comme par exemple la prosopopée, l'allégorie, ou encore des figures qui impliquent une organisation précise peu compatible avec la spontanéité de l'oral telles le chiasme ou certaines formes élaborées de répétitions comme la symploque (Molinié, 1992), l'épanadiplose ${ }^{2}$ ou encore une figure de construction complexe comme le zeugma sémantique.

8 Parmi les autres figures, il semble qu'une distinction soit à faire, bien qu'elle ne puisse avoir une valeur absolue, entre, d'une part, les figures qui correspondent à des faits langagiers caractéristiques de l'oral, et étudiés comme tels dans les ouvrages consacrés à l'oral et, d'autre part, les figures qui ne relèvent de l'oralité qu'en fonction du cotexte.

9 Parmi les figures caractéristiques de l'oral, les figures de type morphologique sont immédiatement identifiables et identifiables comme orales dans la mesure où elles notent des prononciations et où à l'écrit elles entrainent des irrégularités graphiques. Les figures dues à des suppressions courantes à l'oral, telles l'apocope, la syncope et l'aphérèse, sont les plus fréquentes et se différencient par là des figures par adjonction, prosthèse ou épenthèse, qui correspondent à des prononciations considérées comme fautives.

Si l'on considère que la figure suppose une intention, ne faut-il pas considérer comme figures d'autres faits langagiers qui relèvent de la même intention de faire populaire: l'élision de la voyelle dans le pronom de deuxième personne ou dans le pronom relatif sujet ; l'ellipse de l'indice de troisième personne dans les verbes et locutions impersonnels ou celle de la première partie de la négation, ou encore la notation de liaisons qui constituent des adjonctions, que ces liaisons soient fautives (psiloses ou pataquès) ou non ?

11 Les figures de construction qui se signalent immédiatement comme orales sont les figures qui correspondent à une rupture dans le déroulement syntaxique de l'énoncé, c'est-à-dire l'anacoluthe ${ }^{3}$, l'ellipse ${ }^{4}$, l'asyndète ${ }^{5}$, l'aposiopèse ou la réticence qui n'en est qu'une variante ${ }^{6}$. Ce sont aussi les figures de construction qui rendent compte de la constitution de l'oral par ajouts successifs: l'hyperbate, d'une fréquence toute particulière dans le roman contemporain, par laquelle l'énoncé syntaxiquement achevé se prolonge ${ }^{7}$; la polysyndète, qui prolonge l'énoncé grâce à des coordinations successives, mettant ainsi sur l'axe syntagmatique ce qui relève du paradigmatique ${ }^{8}$. 
D'autres figures rendent compte de phénomènes plus spécifiques qui contribuent eux aussi à amplifier ou à prolonger l'énoncé : l'épanorthose sous certaines formes ${ }^{9}$; la paraphrase ${ }^{10}$; le pléonasme ${ }^{11}$ ou encore la réduplication, « répétition littérale, immédiate et iso-fonctionnelle d'un quelconque segment textuel ${ }^{12} »$.

Les figures qui indiquent l'oralité non par elles-mêmes, mais du fait du cotexte, sont surtout les tropes, figures de type sémantique traitées dans les dictionnaires comme emplois figurés de termes appartenant au français standard, qu'il s'agisse de métonymie, de synecdoque ou de métaphore. La métaphore est en général une métaphore in absentia ${ }^{13}$ et opère des recatégorisations banales, dévalorisantes : l'humain devient animal ; l'animé humain devient inanimé : « C'purin-là! » (H. Barbusse, Le Feu (F), 1916) ; l'abstrait devient concret: «Ton anarchisse, tu l'as dans le sang!» $(\mathrm{PQ})$. L'oral ayant volontiers recours à du tout-prêt, peuvent contribuer à l'oralité des tropes d'emploi courant pleinement lexicalisés : «Du reste, il avait du mal à joindre les deux bouts » (O. Adam, Les Lisières (L), 2012). Si la figure ne se dissocie pas d'une motivation ${ }^{14}$, faut-il voir là des figures puisqu'elles peuvent n'être qu'à peine, voire pas du tout, perçues comme telles ? Ainsi n'est-il pas étonnant que les tropes ne soient pas considérés comme suffisants pour caractériser une écriture comme oralisée.

En fait, toute figure est susceptible d'être marque d'oralité si elle est formulée dans un langage non standard. C'est ainsi le cas de figures de diction comme la paronomase : «Ils sont tellement rendus zinzins par les enzymes " (R. Fallet, Le Beaujolais nouveau est arrivé (BN), 1975); de figures de construction comme l'oxymore: «face de dos » (F); ou de figures de pensée comme l'épiphrase: «Il allait presque aussi vite à pied qu'en métro, pour faire ses courses. Alors il mettait le prix du billet dans sa poche. Tout rabiot » (V) ou la litote : « La boîte de veau à la gelée de Mesnil André servirait de hors d'oeuvre. / - Tout ça, ça n'aura rien de sale ! dit Lamuse, ravi » (F).

\section{Les figures de l'oralité : mode d'emploi}

Le mode d'emploi des figures est lié au lieu d'emploi : narration et/ou dialogue.

L'oralité "populaire» restreinte aux dialogues est marquée essentiellement par des figures morphologiques. Celles-ci constituent un véritable code d'écriture du parler populaire (Rouayrenc, 1996). En effet, elles sont figures dans le roman dans la mesure où elles répondent à une intention, celle de faire "populaire", où elles se retrouvent à travers la plupart des romans du début du $\mathrm{xx}^{\mathrm{e}}$ siècle, où certaines de ces marques ont un emploi bien défini, la figure n'apparaissant que dans des conditions limitées (apocope du $e$ caduc utilisée presque exclusivement dans les monosyllabes ${ }^{15}$; alors qu'à l'oral, devant une initiale vocalique, la syncope de è ouvert peut se produire aussi bien dans le déterminant démonstratif que dans "c'est ", dans le roman elle ne concerne à peu près exclusivement que le déterminant et notamment le déterminant masculin : «c't'obus » (F).

17 Une caractéristique de ce code est ainsi de comporter, outre des faits langagiers qui relèvent d'un parler non standard, des faits de niveau liés à la situation socioculturelle du locuteur, des faits qui relèvent du registre oral « courant » si l'on s'en tient à une norme « objective », des faits de registre liés à la situation d'énonciation ${ }^{16}$. Cette forme d'oralité qu'est le parler « populaire » dans le dialogue romanesque, d'une part, met en question la notion de figure (qu'est-ce qui détermine que l'on puisse parler de figure ?) et met en 
évidence l'importance du co(n)texte; d'autre part, oblige à parler de figure que l'on pourrait dire "d'extension restreinte ", restreinte à certains emplois, ce qui précisément fait qu'il y a figure.

18 Le roman contemporain, lui, accorde une bien moindre place à ces figures morphologiques qui néanmoins subsistent dans les dialogues pour indiquer l'oralité, mais sont de fréquence bien moindre et limitées à quelques phénomènes du français oral courant: ainsi dans Les Lisières, outre certains termes apocopés: "appart », "fac» trouve-t-on: la syncope de $"$ bien $»^{17}$, l'ellipse de l'indice de troisième personne et de la première partie de la négation ${ }^{18}$. L'apocope du $e$ dans les monosyllabes ne se rencontre que chez certains écrivains.

19 L'oralité de la narration n'est pas l'oralité des dialogues, en ce qui concerne d'abord les figures morphologiques. Ainsi dans Voyage au bout de la nuit le discours du narrateur comporte $80 \%$ de négations complètes et les dialogues $43 \%$ (Elias, 1981). Dans la narration, la forme «ça » est employée par tous les écrivains, mais ce n'est que chez certains, par exemple Djian dans $37^{\circ} 2$ le matin $\left(37^{\circ}\right)$ (1985), que l'on trouve l'ellipse de «ne» dans la négation. En fait, l'oralité tient essentiellement dans le roman contemporain à des figures de construction.

L'oralité ne peut de toute manière être la même dans le dialogue et dans la narration. Certaines figures sont propres à un mode d'énonciation plutôt qu'à un autre, par exemple l'atténuation, l'aposiopèse au dialogue ${ }^{19}$. La narration autorise plus de développement ${ }^{20}$ et/ou plus d'élaboration, laquelle se traduit par la richesse en figures d'une même phrase ${ }^{21}$. On peut trouver actuellement dans une narration même oralisée des figures qui paraissent peu compatibles avec l'oral, par exemple le chiasme ${ }^{22}$ ou la prosopopée ${ }^{23}$. À travers une figure telle l'hypozeuxe, peuvent se manifester les mêmes recherches rythmiques que dans la langue la plus classique ${ }^{24}$.

21 Il semble ainsi que, même si elle est oralisée, à l'instar du dialogue, la narration garde en quelque sorte la même prééminence par rapport au dialogue que lorsque la langue qui y était employée se différenciait de celle des dialogues.

L'oralité, ce sont aussi les voix dont ces figures sont porteuses, leur dialogisme.

\section{Figures et dialogisme}

J'utilise le terme de "dialogisme» parce que, d'une part, il semble que la voix du narrateur soit toujours prédominante ${ }^{25}$ et, d'autre part, parce que «dialogisme » peut convenir à des énoncés de tout niveau ${ }^{26}$.

\section{La voix de la doxa}

La voix de la doxa s'exprime dans cette parole toute faite, anonyme, que sont les expressions figurées figées. Celles-ci apparaissent bien sûr dans le dialogue, mais aussi dans la narration : «Souvent, [...], les adultes cassaient du sucre sur le dos de Picasso » ( Chagrin d'école (Ch É), 2007). Cette voix s'exprime dans les figures, telle la syllepse, qui créent des jeux de mots attendus : «On lui voyait les côtes [il s'agit d'un chat] comme au bord de la mer » $(\mathrm{F})$; dans les paronomases référant à des formules connues de tous : « On a construit des tours, en hommage au pinard inconnu, au rouquin disparu. [...] Rien que des monuments aux morts! Au Tutu mort, la patrie la dalle en pente !»(BN). La voix de la 
doxa est implicite dans l'hypallage identifiée comme telle en ce qu'elle rompt ce que laisse attendre la doxa pour créer une nouvelle pertinence sémantique, « hyperpertinente ${ }^{27}$ » : « mon frère et ma mère clignotant devant le sapin électrique » (T. Viel, Paris-Brest (PB), 2009). À l'opposé de cette voix collective, identifiable dans toute oralité, se situe la voix du narrateur, individuelle, mais multiple.

\section{Les voix du narrateur}

Le narrateur se fait locuteur dans l'hypotypose qui se traduit dans ce passage par une anaphore : «Comme je les sentais flotter, mes élèves, ces jours-là [...] Mais je n'y suis pas, nom d'un chien, je n'y suis pas, aujourd'hui, je suis ailleurs » (Ch É).

La voix du narrateur peut se doubler de celle d'un métanarrateur, lequel se manifeste essentiellement par la parembole qui rompt le cours de la narration, ce que signale ou non une parenthèse ${ }^{28}$. La parembole est le signe d'un changement énonciatif, d'une voix autre que celle du narrateur. Cette voix s'affirme comme orale quand elle est formulée à la première personne et peut alors être une réflexion suscitée par la narration ${ }^{29}$ ou bien quand une marque intonative révèle le locuteur sous le narrateur : "Son père couché lui en imposait davantage encore que debout sans qu'il pût s'en expliquer les raisons. (Il ne les recherchait d'ailleurs pas!)»(PQ 50) ou encore quand cette voix s'adresse à un destinataire implicite sous la forme d'une interrogation rhétorique : «J'aurais pu lécher son talon aiguille à cet instant ([...] franchement, qui lèche les talons aiguilles de sa fiancée sur un quai de gare ?) » (D. Foenkinos, Les Souvenirs (S), 2011).

Certes, c'est le narrateur qui est censé s'exprimer dans les parties narratives, mais certaines recherches laissent transparaitre une instance supérieure, celle du scripteur qui est l'énonciateur premier (Molinié, 1989: 75). Celui-ci se manifeste (1) dans la distance montrée par les guillemets qui encadrent par exemple les métaphores figées dans les dialogues : « - [...] Elle a l'air "cul bénit" » $(\mathrm{PQ}) ;(2)$ dans les figures strictement orales qui mettent en jeu des récurrences phoniques telles: l'allitération, forme de répétition ${ }^{30}$ : «L'odeur pointue de la poudre et du soufre nous restait comme pour tuer les punaises et les puces de la terre entière" (V); (3) dans la paronomase: "Pas un enfant de la bourgeoisie ne se qualifie ouvertement de bourgeois sans un sentiment confus de honte ontologique» (Ch É); (4) dans la figure dérivative, qui entraine des récurrences phoniques: "Là-bas on entend des cris: Barque est en proie à une ménagerie de ménagères... » $(\mathrm{F})$; (5) dans la déformation paronomastique de certaines expressions figées qui restent implicites : « On perçoit des figures [...] trouées par les veilleuses d'yeux brouillés » $(\mathrm{F}) ;(6)$ dans les manipulations langagières que représentent certaines figures, telle la métaphore "guirlande d'engueulades ", "guirlande " référant à " enguirlander " qui signifie d'abord « entourer de guirlandes" : « à mettons un gosse tous les trois jours, ça en fait une ribambelle de mioches, et une plus belle guirlande d'engueulades encore " $(\mathrm{PQ})$; (7) dans certaines interférences entre instances énonciatives distinctes que seul le scripteur peut maitriser : que ce soit entre narration et dialogue : «On rit. L'homme noir s'en offusqua. Il se leva. / - Vous m'faites mal au ventre, articula-t-il avec mépris. J'vas aux feuillées » (F); «Vous m'faites mal au ventre» du fait du cotexte gauche a un sens figuré et du fait du cotexte droit, le sens propre, ce qui détermine une syllepse ; que ce soit encore entre deux interventions, comme dans ce passage où il n'y a syllepse que pour le lecteur, l'allocutaire textuel ayant interprété l'expression dans son sens figuré : « Là, l' cuistot, c'était le grand Martin César. Il était à la hauteur, lui, pour dégoter du bois. / - Ah! 
oui, lui, c'était un as » (F) ; que ce soit enfin dans l'intertextualité, le scripteur étant le manipulateur qui, à travers certaines figures, fait surgir dans le texte une autre voix, ici, sous l'anaphore, celle de Valéry : "La fesse, rien que la fesse, toujours recommencée » (BN).

\section{Le dialogisme interlocutif}

La figure peut être le biais à travers lequel sont révélées les relations entre personnages d'un roman, relations qui peuvent être de coénonciation (Rabatel, 2012: 96) : ainsi à travers l'utilisation d'une métaphore péjorative figée, Nini, une voisine, adopte, à l'adresse de la femme de Magneux, l'anticléricalisme de celui-ci qui vient de parler d'une « punaise de sacristie » : «- Vous pouvez le plaindre ce sac à charbon!» [prêtre] (PQ) ou de surénonciation - sous-énonciation. La variante « Nous l' savons, nous l' savons ! » (F) de la réduplication courante : «je sais je sais » met le locuteur en position de surénonciation en montrant que celui-ci ne partage pas le même point de vue que son interlocuteur Volpatte dont le long discours précédent dénonçant les embusqués pouvait faire croire à une attitude de surénonciation.

\section{La superposition des voix}

Lorsque le dialogisme est explicite, les voix sont clairement identifiées et identifiables : un locuteur parle avec les mots d'un autre locuteur. C'est ce que l'on rencontre dans Chagrin d'école où le frère du narrateur rapporte les propos de ce dernier lorsqu'il était enfant :

«- [...] Comme j'insistais [...] tu t'es foutu en rogne : «c'est le prof qui l'a dit; les

fractions faut les réduire au dénominateur commun !) »;

qui plus est, ici, la parole du narrateur rapportée au discours direct par son frère qui en est le locuteur a pour énonciateur ${ }^{31}$ le "prof» enfant, ce qui transparait à travers l'apocope « prof » et l'ellipse de l'indice de troisième personne «il » devant « faut ». Il est par ailleurs un autre énonciateur, le scripteur, qui décide de marquer ainsi la voix de l'enfant. On a donc ainsi sous la voix du locuteur trois autres voix : celle de l'enfant, celle du narrateur-enseignant (qui choisit d'interroger son frère: «- [...] quels souvenirs gardes-tu de ma propre nullité, disons ... en math ?») et celle du scripteur. Ailleurs, l'hétérogénéité énonciative est indiquée par un verbe de locution, mais le fragment montré par des guillemets comme parole étrangère est intégré syntaxiquement à la phrase : "On parlait d'un héritage qu'il avait fait, et "ça lui avait tourné le ciboulot" » (PQ).

31 Lorsque le dialogisme est implicite, ce sont des figures indiquant l'oralité qui trahissent sous les propos censés émaner du narrateur le point de vue d'un personnage. Il arrive néanmoins que la parole autre soit suggérée par la présence d'un verbe de locution. La figure significative de l'altérité peut alors être de nature diverse : une expression figée métaphorique $^{32}$, un pléonasme ${ }^{33}$. Souvent aussi le dialogisme n'est décelable qu'aux figures seules: anaphores et parallélismes: "Ça ne servait à rien de discuter, elle ne m'aimait plus c'était tout, elle avait besoin d'air, elle avait besoin d'être libre, elle n'en pouvait plus de me porter à bout de bras depuis tant d'années » $(\mathrm{L})$, ou à des discordances langagières : «Il était pressé de rejoindre sa femme maintenant, vingt cinq ans loin d'elle ça commençait à bien faire » $(\mathrm{L})^{34}$. Ce dialogisme par lequel sont mêlées les voix du 
narrateur et celle des personnages est un des traits d'écriture caractéristiques de bien des romans contemporains ${ }^{35}$.

\section{D'une voix à l'autre}

Les figures peuvent être le pivot favorisant un changement dans l'énonciation. Elles peuvent ainsi indiquer le passage d'une instance énonciative à une autre: voix du narrateur à celle du narrateur narrant. Ou bien la figure indique par elle-même un changement de point de vue, ce qui est le cas de l'épanorthose qui ici permet de faire surgir la voix d'un narrateur narrant à propos de la narration :

«Mais ce fut bien le problème aussi, d'avoir ouvert une boutique pour touristes dans un endroit où il n'y avait pas de touristes. / Non pas qu'il n'y ait pas de touristes dans le Languedoc-Roussillon, je ne dis pas cela, je ne dis même pas qu'il ne devrait pas y avoir de touristes dans le Languedoc-Roussillon, je dis seulement qu'à l'endroit où ma mère a ouvert son magasin pour touristes il n'y avait pas de passage touristique »(P-B).

Ou bien la figure suscite un commentaire. On passe ainsi, à partir d'une comparaison conventionnelle, de la voix du narrateur narré à celle du narrateur-scripteur qui commente à la première personne l'adéquation de l'image à la situation évoquée :

«Son sourire en témoignait largement, auquel je m'accrochai comme à une bouée, je sais, la scène se passe dans une piscine, tant pis, va pour l'image de la bouée, d'autant que dans son regard, juste au-dessus du sourire, je ne me noyais pas, non, c'était bien d'un sauvetage qu'il s'agissait : je me disais, tant que tu la fixes, il ne se passe rien d'irrémédiable » (Ch. Oster, Mon grand appartement, 1999).

Les figures peuvent aussi indiquer le passage d'une modalité énonciative à une autre : de la narration au discours direct, grâce à une anadiplose : «Presque j'ai été content de voir le fils Kermeur qui déjà me tapait sur l'épaule, sur l'épaule de mon vieil ami, a-t-il encore dit devant ma mère outrée » (P-B); d'une formulation indirecte (discours indirect) à une formulation directe (D direct) par le biais par exemple de la réduplication de « $s i$ » : « On s'est dit que cette fois peut-être le gosse comprendrait (D indirect), il s'était bien repris au troisième trimestre (D indirect libre), si, si, je vous assure, il faisait des efforts (D direct libre $\left.{ }^{36}\right) »($ Ch É).

\section{Figures de l'oralité et fonctions}

La fonction et la perception de la fonction de ces figures sont liées au co(n)texte et c'est toujours au lecteur qu'en revient l'actualisation. Une même figure peut avoir des fonctions différentes selon le cotexte ${ }^{37}$ ou cumuler plusieurs fonctions. Des figures différentes peuvent avoir la même fonction ${ }^{38}$. Plutôt que de parler des fonctions qui peuvent être communes aux figures de l'oralité que ce soit dans le dialogue ou dans la narration, la fonction expressive notamment, je m'en tiendrai, faute de place, aux différences de fonctions liées aux différences de lieu d'emploi.

Ainsi tant que toute oralité a été exclue de la narration et que l'oralité « populaire » tenait essentiellement à des figures morphologiques, celles-ci ont eu une fonction sociologique et diégétique. 


\section{Fonction sociologique}

37 On l'a vu, les figures morphologiques ont été utilisées pour marquer l'appartenance à un milieu social, le milieu "populaire». Alors que dans Le pain quotidien la Radigond demande : «- Après... i's'ra pas estropié ?», le médecin répond: «Non... Il ne sera pas estropié Madame... » (PQ). Cette appartenance sociale peut être confirmée par des figures d'une autre nature, notamment les figures que recouvrent les expressions figées : «Vous faites pas d'bile. Laissez l'travail se faire... C'est pas la mer à boire, [...]. » (PQ).

Dans les dialogues du roman contemporain, les figures morphologiques n'ont plus la fonction sociologique qu'elles avaient. Celles qui survivent, déjà indiquées, apparaissent éventuellement quand le dialogue est distinct de la narration et ne marquent que la situation d'énonciation, l'oralité. Elles peuvent éventuellement, en fonction du contexte, être l'indice d'un parler de jeunes : « - Non, pardon, m'sieur, c'est a obtenu. » (Ch É).

Cette fonction sociologique qu'ont pu avoir les figures morphologiques est évidemment inséparable de l'utilisation qui en a été faite dans la construction romanesque, de leur fonction diégétique.

\section{Fonction diégétique}

Ces figures morphologiques ont permis dans le roman du début $\mathrm{du} \mathrm{xx}^{\mathrm{e}}$ siècle une différenciation entre personnages. Dans Voyage au bout de la nuit, les personnages dont le parler est le plus marqué morphologiquement comme oral sont Robinson, Madelon, la vieille Henrouille, Bébert et sa tante chez lesquels 81 \% des négations comportent l'ellipse $\mathrm{du}$ « ne » alors que le parler des médecins ne comporte jamais cette ellipse. Barbusse dans Le Feu fait même du pléonasme la caractéristique langagière du personnage Marthereau : «- C'est curieux et bizarre, [...]. »; «- C'est t'honteux et dégueulasse »; «- C'est vrai et véritable, tout de même »; " - Il est fou et loufoque ». Par ailleurs, au langage est attachée une valeur morale. Chez plusieurs auteurs, T. Rémy, E. Dabit, et notamment chez F. Carco, ce sont les méchants dont le parler se veut le plus populaire ${ }^{39}$. Dans le roman contemporain, à condition de ne pas apparaitre chez d'autres personnages, une figure morphologique peut encore stigmatiser un personnage : ainsi dans «Bille en tête » (1986) de A. Jardin, un proviseur, maniaque sexuel : «Vot' mère vient d'arriver de Paris. Elle veut vous voir tout de fuite $»$.

\section{Fonction stylistique}

Chez certains écrivains contemporains, l'oralité relève de la fonction poétique telle que l'entend R. Jakobson (1963: 218) ${ }^{40}$. L'oralité se voulant alors style, je parlerai de fonction stylistique. On ne semble pouvoir parler de fonction stylistique que dans la mesure où la recherche de l'expression de l'oralité est le but de l'écriture, ce qui ne peut être obtenu véritablement quand l'oralité se réduit à l'addition de traits oraux ${ }^{41}:$ "Y avait une fille qui dansait à moitié à poil sur l'étiquette, une plage déserte avec de l'eau bleu, les types s'étaient pas cassé le citron " $\left(37^{\circ}\right)$. Mais dès lors qu'il y a cohésion dans l'écriture ${ }^{42}$ et travail de transposition ${ }^{43}$, ne peut-on parler d'une littérarité de l'oralité à laquelle participent les figures vues ? En fait, c'est le cotexte oralisé qui fait de certaines figures, notamment des figures de construction, des figures de l'oralité. 
42 À travers l'emploi qui est fait de celles-ci, l'écriture contemporaine semble être peu ou prou une écriture que l'on pourrait dire oxymorique dans la mesure où elle tente de concilier des opposés, continuité et discontinuité d'abord, du fait de la syntaxe et/ou de la ponctuation, et ce de manière quelque peu différente selon qu'il s'agit d'asyndètes, d'ellipses, d'anacoluthes ou d'hyperbates.

L'écriture contemporaine tente aussi de concilier axe syntagmatique, sur lequel se réalise une progression syntaxique et sémantique et axe paradigmatique qui ralentit cette progression, et ce selon deux modalités: une modalité verticale par laquelle prime le paradigmatique grâce ici à l'épitrochasme :

«Le vent les marées le sable la roche et le ciel me reconnectaient au vivant [...].»

(L) ou à des parallélismes que peut souligner une épanaphore: «Je n'en revenais

pas. Que les choses aillent si vite. Que ma mère n'ait même pas visité. Que mon père

ait pris cette décision. Qu'il abandonne la maison comme ça, en un clin d'oeil » (L) ;

une modalité à la fois verticale et horizontale qui combine insistance, approfondissement et progression, grâce notamment à l'anadiplose : «Il a une femme et trois enfants, une femme excessive quand on connaît Istvan » (A.-M. Garat, Istvan arrive par le train du soir, 1999). À cela peut être ajouté un oxymore énonciatif chez bon nombre d'écrivains, la narration intégrant sans différenciation une autre modalité énonciative : «On peut rester amis, non ? [...] Elle me dit que non, vraiment, qu'il vaut mieux qu'on arrête de se voir " (O. Adam, À l'ouest, 2001), parfois jusqu'à la syllepse énonciative. La superposition de la voix du narrateur et de celle du personnage est en effet fréquente et se fait en général de la même manière : la superposition des voix est en quelque sorte créée et explicitée après coup par une phrase ou une incise qui en indique l'énonciateur sous-jacent:

« C'est pour ça qu'elle était particulièrement gentille et attentive avec moi, ce Noël-

là, et c'est pour ça qu'en même temps elle était inquiète, parce qu'avec moi on ne peut jamais savoir, pensait-elle, que j'étais un intellectuel et qu'en tant qu'intellectuel je me croyais tout permis » (P-B).

le second « avec moi » qui ne peut référer qu'au seul narrateur est présenté comme étant aussi la pensée du personnage.

En fait, n'y a-t-il pas un oxymore d'un autre niveau, cette écriture alliant analyse et émotion?

«J'avais beau me frotter les yeux, on se battait désormais pour vivre dans cet endroit où j'avais grandi, que j'avais toujours voulu quitter, que j'avais voulu voir mes parents quitter à leur tour. Qu'est-ce qui les retenait ici ? Quel attachement? Quelles racines? Quelle inertie?» (L)

47 Analyse soulignée ici par le parallélisme des relatives, l'anaphore de «j'avais voulu » et de "quitter », par le parallélisme des syntagmes nominaux interrogatifs, et émotion par la figure du paradoxe et par l'épanaphore « quel(les)».

\section{Conclusion}

En un siècle, l'oralité a beaucoup changé... de figures notamment et de lieu d'emploi. Cantonnée d'abord au dialogue, elle a ensuite progressivement tout envahi. Est-ce à dire que l'oralité est la même dans les deux formes d'énonciation? Certes non. Glissant dans la narration et n'ayant plus la même finalité, l'oralité se fonde essentiellement sur les figures de construction caractéristiques de l'oral. Le dialogue, étant moins nettement différencié de la narration et ce d'autant plus que le discours direct est souvent intégré 
dans la narration, se vide des figures qui le caractérisaient. La narration garde ainsi sa prééminence, restant le lieu où apparait à plein le travail de l'écriture, écriture de la discontinuité, de l'absurde du fait de l'asyndète omniprésente, écriture de l'approfondissement par retouches successives du fait des anaphores sous leurs diverses formes. L'oralité peut actuellement faire la littérarité d'un texte, son épaisseur tenant au mélange des voix.

Toutefois, n'est-on pas passé finalement, en oralisant la narration, d'un code "populaire» du dialogue à un code d'oralité « littéraire», ou littéraire d'oralité, de la narration dans la mesure où d'un roman à l'autre, même si l'on peut observer des prédominances, on retrouve une oralité fondée sur les mêmes figures fondamentales, quelque peu contradictoires : l'anaphore, l'hyperbate et l'asyndète?

\section{BIBLIOGRAPHIE}

ADAM, J.-M. (1994) : «Style et fait de style : un exemple rimbaldien », in : G. Molinié \& P. Cahné (dirs), Qu'est-ce que le style, Paris, Presses universitaires de France, p. 15-43.

BÉDARD, É., MAURAIS, J. (éds) (1983) : La Norme linguistique, Paris, Le Robert.

BLANCHE-BENVENISTE, C. (1997): Approches de la langue parlée en français, Gap-Paris, Ophrys.

воNномме, M. (1998) : Les Figures clés du discours, Paris, Éd. Le Seuil.

- (2005) : Pragmatique des figures du discours, Paris, H. Champion.

BRES, J. (2005) : « Savoir de quoi on parle : dialogue, dialogal, dialogique ; dialogisme, polyphonie...», in : J. Bres, P. P. Haillet, S. Mellet, H. Nølke \& L. Rosier (dirs), Dialogisme et polyphonie Approches linguistiques. Actes du colloque de Cerisy, Bruxelles, De Boeck, p. 47-61.

CAHNÉ, P. (1994) : « Qu'est-ce que la forme ? », in : G. Molinié \& P. Cahné (dirs), Qu'est-ce que le style, Paris, Presses universitaires de France, p. 63-69.

CAlAS, F., FROMilHAGUe, C., GARAGNON, A.-M. \& SUSini, L. (dirs) (2012) : Les Figures à l'épreuve du discours. Dialogisme et polyphonie, Paris, Presses de l'Université Paris-Sorbonne.

DERVAL, A. (éd.) (1993) : Voyage au bout de la nuit de Louis-Ferdinand Céline Critiques 1932-1935, Saint-Germain la Blanche-Herbe, Éd. de l'Imec.

DU MARSAIS, C. C. (1977) [1730] : Traité des tropes, Paris, Éd. Le Nouveau Commerce.

DUCROT, O. (1984) : Le Dire et le dit, Paris, Éd. de Minuit.

DUPRIEZ, B. (1980) : Gradus. Les procédés littéraires (dictionnaire), Paris, Union générale d'éditions.

ELIAS, B. (1981) : «Personnages et idiolectes dans Voyage au bout de la nuit et Nord », in : Actes du colloque international d'Oxford, 13-16 juil., Bibliothèque Céline de l'Université Paris VII, p. 253-267. FROMilhague, C. (1995) : Les Figures de style, Paris, Nathan.

GAUDIN, L. \& SALVAN, G. (2008) : « Le sens en marche : le cas de l'hypallage », L'Information grammaticale, 116, p. 18-19. 
- (2010) : « De la non-pertinence à l'hyperpertinence : Intrig(u)antes figures dans Voyage au bout de la nuit », in: Au Corps du texte, Paris, H. Champion, p. 277-293.

JAKOBSON, R. (1963) : Essais de linguistique générale, Paris, Éd. de Minuit.

JAUBERT, A. (2012) : «Le processus énonciatif de l'acte figural », in : F. Calas, C. Fromilhague, A.M. Garagnon \& L. Susini (dirs), Les Figures à l'épreuve du discours. Dialogisme et polyphonie, Paris, Presses de l'Université Paris-Sorbonne, p. 31-38.

JOLLIN-BERTOCCHI, S. (2003) : Les Niveaux de langage, Paris, Hachette.

Le Français moderne, 1 (1982) : « La norme concept sociolinguistique ».

LÉON, P. R. (1971) : Essais de phonostylistique, Montréal, M. Didier.

MAINGUENEAU, D. (1994) : «L'horizon du style », in: G. Molinié \& P. Cahné (dirs), Qu'est-ce que le style, Paris, Presses universitaires de France, p. 187-199.

MOLINIÉ, G. (1989) : La Stylistique, Paris, Presses universitaires de France.

- 1992, Dictionnaire de rhétorique, Librairie générale française.

MORIER, H. (1975) [1961] : Dictionnaire de poétique et de rhétorique, Paris, Presses universitaires de France.

PHILIPPE, G. \& PIAT, J. (dirs) (2009) : La Langue littéraire. Une histoire de la prose en France de Gustave Flaubert à Claude Simon, Paris, Fayard.

RABATEL, A. (2012) : « Les postures énonciatives dans la co-construction dialogique des points de vue : coénonciation, surénonciation, sous-énonciation » in : J. Bres, P. P. Haillet, S. Mellet, H. Nølke \& L. Rosier (dirs), Dialogisme et polyphonie Approches linguistiques. Actes du colloque de Cerisy, Bruxelles, De Boeck, p. 95-110.

ROSIER, L. (1999) : Le Discours rapporté. Histoire, théories, pratiques, Bruxelles, De Boeck.

ROUAYRENC, C. (1988) : Recherches sur le langage populaire et argotique dans le roman français de 1914 à 1939, thèse, Université Paris III.

- (1994) : «C'est mon secret » La technique de l'écriture "populaire" dans Voyage au bout de la nuit et Mort à crédit, Tusson, Du Lérot.

- (1996) : «Le parlé dans le roman : variations autour d'un code », Versants, 30, pp. 31-44

WATINE, M.-A. (2012) : « La réduplication : une interprétation dialogique », in : F. Calas,

C. Fromilhague, A.-M. Garagnon \& L. Susini (dirs), Les figures à l'épreuve du discours. Dialogisme et polyphonie, Presses de l'Université Paris-Sorbonne, p. 149-164.

WOLF, N. (1990) : Le Peuple dans le roman français de Zola à Céline, Paris, Presses universitaires de France.

\section{NOTES}

1. Préface de Germinie Lacerteux (1865).

2. Entendue comme la définit C. C. Du Marsais «Lorsque de deux propositions corrélatives, l'une commence et l'autre finit par le même mot. » : voir B. Dupriez (1980 : 187).

3. " Il y a ceux qui se suicideront après et ça ne se voit pas sur leur visage » (D. Pennac, La Fée Carabine, 1987).

4. «Bien sûr le temps passerait, bien sûr la croissance, bien sûr les événements, bien sûr la vie »

(D. Pennac, Chagrin d'école, 2007). 
5. « Ma dernière visite avait été douloureuse, je n'avais presque pas reconnu ma mère » (D. Foenkinos, Les Souvenirs, 2011).

6. «À vrai dire, aucune de ces explications n'est absolument satisfaisante. Toutes tiennent plus ou moins, mais...» (Ch É).

7. «Il en imposait. Même à moi, qui n'avais jamais réussi à ne pas me sentir rabaissé face à un mec comme lui » (O. Adam, Les Lisières, 2012).

8. « Moi-même je sais où mon La Bruyère m'attend, je le vois sur son étagère et mon Conrad, et mon Lermontov, et mon Perros, et mon Chandler... » (Ch É).

9. «Son souvenir je le saisis, pas lui bien sûr, mais le souvenir précisément de ce Robinson » (V).

10. «Parce qu'elle je m'en moquais, je m'en foutais vraiment de ça, d'elle» (L. Mauvignier, Apprendre à finir, 2000).

11. «J'sais bien qu'un violon, ça sert pas à grand'chose pour l'utilité, mais tout d'même... » (H. Barbusse, Le Feu, 1916).

12. « Et surtout, surtout, je hais cette peur du pauvre » (Ch É). Voir M.-A. Watine (2012).

13. «- Ça de cric pour douze hommes, la veille d'une attaque!» (R. Dorgelès, Les Croix de bois, 1919).

14. Voir A. Jaubert (2012 : 35) «Une figure est perçue comme une figure lorsqu'elle se révèle en congruence avec une intentionnalité. »

15. Voir P. R. Léon (1971: 78) : «Lorsqu'on veut faire familier ou vulgaire, il suffit de remplacer les e caducs par des apostrophes ».

16. Voir C. Rouayrenc (1994). S. Jollin-Bertocchi (2003: 37-47) parle pour les premiers d'« axe vertical » et pour les seconds d'« axe horizontal ».

17. «- Ben toi t'étais déjà un rêveur. » (L).

18. « - Où t'as pu traîner. Y a rien. » (ibid.)

19. «Alors qu'est-ce qu'il voulait? elle a insisté. / - Rien... Des conneries... Il veut qu'on repeigne deux ou trois trucs [en fait repeindre toutes les "baraques" d'un motel]» $\left(37^{\circ}\right)$.

20. " Notre époque s'est fait un devoir de jeunesse : il faut être jeune, penser jeune, consommer jeune, vieillir jeune (oxymore), la mode est jeune, le foot est jeune, les radios sont jeunes, les magazines sont jeunes, la pub est jeune, la télé est pleine de jeunes (antanaclase), internet est jeune, les people sont jeunes, les derniers baby boomers vivants ont su rester jeunes, nos hommes politiques eux-mêmes ont fini par rajeunir. Vive la jeunesse ! (figure dérivative) Gloire à la jeunesse ! il faut être jeune !» (Ch É).

21. Ainsi dans : «Une de ces émissions défouloirs où les auditeurs ont la parole, où les auditeurs commentent l'actualité, où les auditeurs commentent les commentaires des auditeurs qui ont commenté l'actualité » (S) se rencontrent : une figure dérivative : (commentent, commentaires), un polyptote (commentent, ont commenté), une hypozeuxe (où les auditeurs...où les auditeurs.... où les auditeurs).

22. «Je suis retourné [...] là où on peut se retrouver sur la dune à regarder les mouettes et la mer ce jour là plutôt calme. Tellement calme ce jour-là que depuis la route on pouvait voir l'île d'Ouessant contredire l'horizon» (T. Viel, Paris-Brest).

23. «C'est Mère-Grand marketing qui habille grands et petits. [...] quand il [l'élève] s'assied en classe, c'est elle qui vibre au fond de sa poche pour le rassurer : Je suis là, n'aie pas peur, je suis là, dans ton téléphone » (Ch É).

24. Ainsi une période en cadence mineure: «Le jour où François avait voulu le convaincre de décorer un peu l'endroit, de poser des jolis rideaux, d'acheter une lampe avec un bel abat-jour pour ne plus utiliser cet affreux néon, d'accrocher des cadres, de couvrir ses draps d'un dessus-de lit, d'acheter une nouvelle commode, il avait refusé net » (L).

25. «La polyphonie étant réservée aux cas d'hétérogénéité énonciative sans hiérarchisation des voix » (Jaubert, $2012: 33$ ). 
26. «Si le dialogisme est à situer d'abord au niveau macro de l'énoncé-texte, il est bien évident qu'il se manifeste aux différents niveaux inférieurs qui composent cette unité, notamment celui des énoncés-phrases, ou, celui, encore inférieur des mots eux-mêmes, ce qu'avait bien vu Bakhtine » (Bres, $2005: 60)$.

27. "L'hypallage donne toujours à entendre le stéréotype, ou la doxa. Si l'attendu (linguistique, moral ou discursif) ne s'entend pas, il n'y a pas hypallage » (Gaudin \& Salvan, 2008). Voir aussi L. Gaudin-Bordes et G. Salvan (2010).

28. «(“N'est-ce pas qu'il faut savoir rester jeune ?”) » (Ch É) ; « C'est ce que font tous les hommes qui viennent de devenir père, non?» $(\mathrm{S})$.

29. "Je progresse à tâtons dans cet intérieur obscur, encombré de meubles anguleux et d'objets hideux (je le devine)» (É. Chevillard, Démolir Nisard, Paris, Éditions de Minuit, 2006).

30. C. Fromilhague (1995: 23) parle de figures de « continuité phonique ».

31. "J'appelle "énonciateurs" ces êtres qui sont censés s'exprimer à travers l'énonciation, sans que pour autant on leur attribue des mots précis » (Ducrot, 1984 : 204).

32. «Elle lui avait dit [...] qu'elle était navrée [...] de voir l'air aimable de porte de prison, dont Nini avait l'intelligence de ne pas se formaliser » $(\mathrm{PQ})$.

33. " Entre deux grosses roches il s'était établi une sorte de cagna, à l'abri, me fit-il remarquer, des tornades de l'Est, les plus mauvaises, les plus rageuses » (V).

34. Dans Ravel, c'est par cet effet de contraste que J. Echenoz introduit dans la narration le point de vue du personnage : ici par une métaphore figée et l'ellipse du verbe : « Il traîne, il a un mal de chien, pas moyen de trouver comment l'[le concerto] achever. »

35. "Soudain grand-père allume la lumière et nous engueule parce que c'est l'heure de dormir et que la propriétaire habite à l'étage d'en dessous alors faut pas faire de bruit » (O. Adam, À l'ouest, 2001).

36. Voir L. Rosier (1999 : 266-291).

37. La réduplication (en fait triplication) peut exprimer un désir obstiné chez le locuteur (fonction expressive) : «Elle disait : 〈Je veux rester chez moi, je veux rester chez moi, je veux rester chez moi.)» (S 32) ; ailleurs elle peut être un outil de persuasion (fonction conative) : «Vous croyez que vous ne savez rien, mais vous vous trompez, vous vous trompez, vous en savez énormément! »(Ch É).

38. L'ironie peut ainsi s'exprimer par l'hyperbole: «Il [le député] a dit que Mme Vivieux [centenaire] connaissait certainement par cœur tout ce merveilleux répertoire de nos belles chansons françaises» (M.-S. Roger, Les Encombrants, 2007), et par l'anaphore pléonastique de «tous » et de « chacun » et le paradoxe qui suit, dans la dernière partie de ce passage: « M. le député-maire a parlé de la fraternité de tous envers les personnes âgées [...] et de l'égalité de chacun devant la vieillesse, tant qu'on a la santé, et de cette liberté de tous et de chacun de mourir loin des siens, en maison de retraite » ( M.-S. Roger, ibid.).

39. Dans La Rue (1930) de F. Carco, le personnage de Maurice, dont on apprend par la suite qu'il a commis un meurtre, est celui dont le parler, caractérisé dès le début du roman, est tout au long du roman le plus marqué comme populaire.

40. «La visée du message en tant que tel, l'accent mis sur le message pour son propre compte est ce qui caractérise la fonction poétique du langage ».

41. «Le style est bien dans les détails, mais dans tous les détails, et dans toutes leurs relations »

(G. Genette cité in : Maingueneau, 1994 : 197).

42. Voir J.-M. Adam (1994: 27) et P. Cahné (1994: 63-69).

43. Voir Céline : Image du bâton dans l'eau, Cahiers Céline 2, Paris, Gallimard, 1976, p. 87 ; image des rails, Entretiens avec le Professeur Y, Paris, Gallimard, 1955, pp. 110-111. 


\section{RÉSUMÉS}

L'oralité est co(n)textuelle et a pu varier selon son domaine d'emploi : ou bien, au début du $\mathrm{xx}^{\mathrm{e}}$ siècle, le dialogue seul, ce qui la faisait percevoir comme langage populaire, par opposition au langage littéraire de règle dans la narration, ou bien le dialogue et la narration. Les figures de l'oralité dans le roman sont d'une part des figures, morphologiques et syntaxiques, qui se caractérisent d'emblée comme orales, d'autre part des figures, les tropes notamment, dont l'oralité tient au cotexte. L'oralité, ce sont aussi les voix diverses qui s'expriment à travers les figures. Le langage perçu comme populaire tient pour l'essentiel à des figures morphologiques dont l'emploi est codifié et dont la fonction est sociologique et diégétique. L'extension de l'oralité n'a pas entrainé l'uniformisation que l'on pourrait attendre entre narration et dialogue. Dans le roman contemporain, l'oralité tient surtout à des figures de construction et acquiert une fonction stylistique de nature oxymorique qui conserve au langage de la narration la prééminence sur celui des dialogues. Toutefois, l'oralité, de code «populaire » qu'elle était dans le dialogue n'est-elle pas devenue code « littéraire » dans la narration, où, d'un roman à l'autre, elle semble fondée sur quelques mêmes figures?

Orality, being co(n)textual, may have varied according as it was used in dialogue alone, at the beginning of the 20th century, which made it appear as popular language, as opposed to literary language prevailing in narration, or in dialogue and narration. The figures of orality in the novel are, on one hand, figures belonging to morphology or to syntax that are straightaway perceived as oral; on the other hand figures, mainly tropes, the orality of which comes from the cotext. Orality also consists of the various voices that express themselves through figures. The characteristic of language perceveid as popular comes mainly from morphological figures the use of which is codified, their functions being sociological and diegetic. The spreading use of orality hasn't led to uniformity between narration and dialogue as might have been expected. In contemporary novel, orality stems from construction figures and assumes a stylistic function of an oxymoristic nature thanks to which the language of narration prevails over that of dialogues. Nevertheless, hasn't orality, from the "popular" code it used to be in dialogue, become a "literary" code in narration, in which, from novel to novel, it seems to be based on some identical figures?

\section{INDEX}

Mots-clés : oralité, figures, dialogisme, narrateur, scripteur, « populaire » (langage)

Keywords : orality, figurs, dialogism, narrator, scriptor, "popular" (language)

\section{AUTEUR}

\section{CATHERINE ROUAYRENC}

Université de Toulouse-Le Mirail 